\title{
РЕАЛИЗАЦИЯ ФОТОЭЛЕКТРИЧЕСКОГО ЭФФЕКТА 2D ГРАФЕНОВЫХ СТРУКТУР ДЛЯ ИНИЦИИРОВАНИЯ ВЗРЫВЧАТОГО РАЗЛОЖЕНИЯ КОМПЛЕКСНОГО ПЕРХЛОРАТА КОБАЛЬТА (III)
}

\author{
М.А. Илюшин ${ }^{1}$, Ю.Н. Ведерников ${ }^{2}$, А.П. Возняковский ${ }^{3}$, И.В. Шугалей ${ }^{1}$, А.В. Смирнов ${ }^{2}$, \\ А.И. Коваленко ${ }^{2}$, В.Г. Бутенко ${ }^{4}$, Ю.А. Кулагин ${ }^{5}$ \\ ${ }^{1}$ Санкт-Петербургский государственный технологический институт (технический университет) \\ (СПбГТИ(ТУ), пр-т Московский, 26, Санкт-Петербург, Российская Федерация, 190013 \\ E-mail: explaser1945@yandex.ru \\ ${ }^{2}$ Акционерное Общество «Научно-производственное предприятие «Краснознамёнец», ул. Челябинская, \\ 95, Санкт-Петербург, Российская Федерация, 195043 \\ E-mail: vedjrnik@mail.ru,smirnov157@yandex.ru, terranova163@gmail.com \\ ${ }^{3}$ Научно-исследовательский институт синтетического каучука им. С.В. Лебедева, ул. Гапсальская, 1 , \\ Санкт-Петербург, Российская Федерация, 198035 \\ E-mail: voznap@mail.ru \\ ${ }^{4}$ Федеральное государственное бюджетное учреждение науки Межведомственный центр аналитических \\ исследований в области физики, химии и биологии при Президиуме Российской академии наук (МЦАИ \\ РАН), ул. Профсоюзная, 65, с.6, г. Москва, Российская Федерация, 117342 \\ E-mail: cnfc2009@mail.ru \\ ${ }_{5}^{5}$ Государственный научный центр Российской Федерации Федеральное государственное унитарное \\ предприятие «Центральный научно-исследовательский институт химии и механики» (ГНЦ РФ ФГУП \\ «ЦНИИХМ»), ул. Нагатинская, д. 16а, г. Москва, 115487 \\ E-mail: kulaginyua@mail.ru
}

Модификация поверхности кристаллов энергетических материалов (ЭМ) наноуглеродными добавками представляет собой эффективный путь целенаправленного повышения их восприимчивости к электромагнитному излучению. В качестве ЭМ для последующей модификации был использован комплексный перхлорат кобальта (III) - перхлорат пентааммин(5-нитротетразолато-N2) кобальта (III) (NCP). Наноуглеродной добавкой служили многослойные (2-5 слоев) 2D графеновые листы (GnS), содержсащие терминальные гидроксильные группы. Источником когерентного излучения являлся лазерный диод (ЛД) с длиной волны 976 нм. Излучение от ЛД передавали на мичень через оптическое волокно. Было приготовлено и испытано 2 серии образцов: пиротехническая смесь, содержащая 97\% NCP и 3\% GnS, и комплекс NCP. Отдельными опытами было найдено, что при смешении комплекса NCP и графена GnS в ультразвуковом поле происходит взаимодействие между компонентами смеси. Эксперименты с ЛД показали, что пиротехническая смесь NCP c GnS имеет порог восприимчивости клучу лазерного диода примерно 200 раз ниже, чем комплекс NCP той эже дисперсности. Высказана гипотеза, не противоречащая полученным экспериментальным данным, что инициирование пиротехнического состава NCP/GnS происходит вследствие возникновения фототока в графеновой добавке под воздействием когерентного лазерного излучения (явление фотовольтаики). Проведены испытания пиротехнического состава NCP/GnS 97/3\% в макете лазерного капсюлядетонатора с положительными результатами. Результаты работы могут служить основой для разработки безопасных, надежных и экологичных лазерных детонаторов для применения в горнодобывающей, нефтегазовой промышленности и других отраслях народного хозяйства.

Ключевые слова: лазерное инициирование, многослойный графен, комплексный перхлорат кобальта (III) 


\title{
2D GRAPHENE STRUCTURES PHOTOELECTRIC EFFECT ON INITIATION OF COBALT (III) PERCHLORATE COMPLEX EXPLOSIVE DECOMPOSITION
}

\author{
M.A. Ilyushin ${ }^{1}$, Yu.N. Vedernikov ${ }^{2}$, A.P. Voznyakovskii ${ }^{3}$, I.V. Shugalei ${ }^{1}$, A.V. Smirnov ${ }^{2}$, \\ A.I. Kovalenko ${ }^{2}$, V.G. Butenko ${ }^{4}$, Yu.A. Kulagin ${ }^{5}$ \\ ${ }^{1}$ Saint-Petersburg State Institute of Technology (Technical University), Moskovsky Ave., 26, Saint-Petersburg, \\ Russian Federation, 190013 \\ E-mail: explaser1945@yandex.ru \\ ${ }^{2}$ Joint Stock Company "Research and Production Enterprise" Krasnoznamenets ", st. Chelyabinskaya, 95 , \\ Saint-Petersburg, Russian Federation, 195043 \\ E-mail: vedjrnik@mail.ru, smirnov157@yandex.ru, terranova163@gmail.com \\ ${ }^{3}$ Research Institute of Synthetic Rubber named after S.V. Lebedev, st. Gapsalskaya, 1, Saint-Petersburg, Russian \\ Federation, 198035 \\ E-mail: voznap@mail.ru \\ ${ }^{4}$ Federal State State-financed Institution of Sciences Interdepartmental Center of Analytic Studies in the Sphere \\ of Physics, Chemistry and Biology under Presidium of Russian Academy of Sciences (ICAS RAS), 65 Trade \\ Union Street, p. 6, Moscow, Russian Federation, 117342 \\ E-mail: cnfc2009@mail.ru \\ ${ }^{5}$ The Central Scientific Research Institute of Chemistry and Mechanics, Nagatinskaya str., 16a, Moscow, \\ Russian Federation, 115487 \\ E-mail: kulaginyua@mail.ru
}

Modification of energetic materials (EM) surface crystals with nanocarbon additives is an effective way to increase their susceptibility to electromagnetic radiation. Complex cobalt (III) perchlorate - pentaammine (5-nitrotetrazolato-N2) cobalt (III) perchlorate (NCP) was used as an EM for the subsequent modification. Multilayer (2-5 layers) $2 D$ graphene sheets (GnS) containing terminal hydroxyl groups served as a nanocarbon additive. The laser diode $(L D)$ with a wavelength of $976 \mathrm{~nm}$ was used as the source of coherent radiation was. Radiation pulse from the LD was transmitted to the target through an optical fiber. Two series of samples: a pyrotechnic mixture containing 97\% NCP and 3\% GnS, and an NCP complex were prepared and tested. Separate experiments have shown that mixing of the NCP complex and graphene GnS in an ultrasonic field leads to interaction between the components of the mixture. Experiments with LD have fixed that a pyrotechnic mixture of NCP with GnS has a threshold of susceptibility to a laser diode beam about 200 times lower than an NCP complex of the same dispersion. There was made an assumption that the initiation of the pyrotechnic composition NCP/GnS occurs due to the appearance of a photocurrent in the graphene additive under the influence of coherent laser radiation (the phenomenon of photovoltaics). This assumption doesn't contradict with obtained experimental date. The pyrotechnic composition NCP/GnS 97/3\% was tested in a model of a laser blasting cap with positive results. The results of present work may form a basis of the development of safe, reliable and environmentally friendly laser detonators for use in the mining, oil and gas industry and other sectors of the national economy.

Key words: laser initiation, multilayer graphene, complex cobalt (III) perchlorate

Для цитирования:

Илюшин М.А., Ведерников Ю.Н., Возняковский А.П., Шугалей И.В., Смирнов А.В., Коваленко А.И., Бутенко В.Г., Кулагин Ю.А. Реализация фотоэлектрического эффекта $2 \mathrm{~d}$ графеновых структур для инициирования взрывчатого разложения комплексного перхлората кобальта (III). Рос. хим. ж. (Ж. Рос. хим. об-ва). 2021. T. LXV. № 3. С. 19-24

For citation:

Ilyushin M.A., Vedernikov Yu.N., Voznyakovskii A.P., Shugalei I.V., Smirnov A.V., Kovalenko A.I., Butenko V.G., Kulagin Yu.A. 2D graphene structures photoelectric effect on initiation of cobalt (III) perchlorate complex explosive decomposition. Ros. Khim. Zh. 2021. V. 65. N 3. P. 19-24 


\section{ВВЕДЕНИЕ}

Модификация поверхности кристаллов энергетических материалов (ЭМ) - эффективный путь изменения их целевых свойств. Особый интерес в качестве объектов модификации представляют энергонасыщенные металлокомплексы, применяемые в качестве компонентов систем, разлагающихся по механизму разветвленных цепных реакций под действием энергетических импульсов [1-3]. Перспективность применения энергонасыщенных металлокомплексов в промышленности обусловлена их меньшей токсичностью по сравнению с традиционно используемыми энергонасыщенными солями, такими, как фульминат ртути, азид свинца или стифнат свинца $[4,5]$.

Предполагается, что модификация поверхности ЭМ наноуглеродными (НУ) добавками должна изменить их восприимчивость к внешнему воздействию. В качестве ЭМ для последующей модификации был выбран комплексный перхлорат кобальта (III) - перхлорат пентааммин(5-нитротетразолато- $\mathrm{N}^{2}$ ) кобальта(III) (NCP) (см. рис. 1), предложенный в качестве основного заряда в преобразователе взрывного процесса, используемого в аппаратуре для перфорации глубоких нефтяных и газовых скважин [6]. Так, из литературы известно, что добавки детонационных наноалмазов (ДНА), фуллеритов $\mathrm{C}_{60}$ (кристаллов фуллерена $\mathrm{C}_{60}$ ) и $2 \mathrm{D}$ многослойных графеновых нанолистов $(G n S)$, содержащих терминальные гидроксильные группы, которые получены методом самоподдерживающегося высокотемпературного синтеза при карбонизации смеси крахмала и нитрата аммония - повышает восприимчивость энергонасыщенного металлокомплекса NCP к действию сильноточного пучка электронов [7-9]. В смесях с НУ добавками возрастала эффективность воздействия продуктов взрыва комплекса NCP на преграду, характеризуемая глубиной отпечатка на металлической пластине-свидетеле. При этом эффективность НУ добавок увеличивалась в ряду ДНA $<\mathrm{C}_{60}<G n S$. Также было показано, что добавка небольшого количества оксида графена привела к снижению порога зажигания лазерным излучением нитроцеллюлозы и гексанитрогексаазаизовюрцитана (CL-20) [10-12].

Мы предположили, что экспериментально показанное различное влияние морфометрических параметров частиц наноуглерода на эффективность инициирования ЭМ сильноточным пучком электронов и лазерным лучом обусловлено изменением эффективности доставки электронов частицами наноуглерода к частицам ЭМ. Выдвинутое предположение было экспериментально проверено в представленной работе на примере влияния добавки GnS [13] на чувствительность комплекса $\mathrm{NCP}$ воздействию луча лазерного диода.

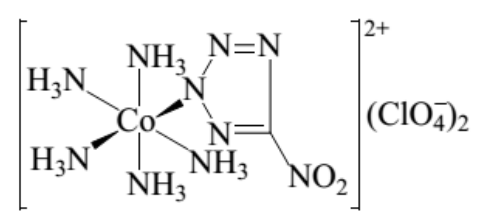

Рис. 1. Структурная формула перхлората пентааммин(5-нитротетразолато- $\mathrm{N}^{2}$ ) кобальта (III) (NCP)

\section{МЕТОДИКА ЭКСПЕРИМЕНТА}

Синтез комплекса. Комплекс NСР был получен при взаимодействии перхлората аквапетааммината кобальта с 5-нитротетразолом в среде разбавленной хлорной кислоты в течение трех часов при повышенной температуре [6]. Свойства полученного образца комплекса NCP соответствовали приведенного в работах $[4,14]$.

Синтез 2D наноуглерода. Графеновые нанолисты $\mathrm{GnS}$ получены методом самораспространяющегося высокотемпературного синтеза (CBC) в волне горения смеси нитрата аммония и крахмала в весовом соотношении 1:1 [13].

Особенности получения смеси NCP c GnS. Смешение комплекса NCP c GnS производили путем их облучения ультразвуком мощностью 50 Вт в ванне «Сапфир» в среде толуола в течение 60 мин. После смешения толуол испаряли при комнатной температуре до отсутствия запаха. Остаток толуола удаляли путем сушки при температуре 80 ${ }^{\circ} \mathrm{C}$ в течение 60 мин.

\section{РЕЗУЛЬТАТЫ И ИХ ОБСУЖДЕНИЕ}

В представленной работе было изучено воздействие излучения лазерного диода с длиной волны 976 нм (область оптической прозрачности матрицы) как на заряд комплекса NCP, так и на пиротехническую смесь $97 \% \mathrm{NCP}+3 \% \mathrm{GnS}$. Излучение лазерного диода передавали на мишень через оптическое волокно с диаметром сердцевины 105 мкм с числовой апертурой 0,15 . Регистрация вспышки состава осуществлялась с помощью регистрирующего световода, подключенного к фотодиоду. Фотодиод генерировал электрический сигнал от вспышки продуктов детонации. Запуск осциллографа осуществляли от подачи лазерного излучения или непосредственно от фотодиода, срабатывающего от отраженного света инициирующего световода.

На рис. 2 приведена осциллограмма процесса лазерного инициирования макета лазерного 
капсюля-детонатора (ЛКД), содержащего заряд светочувствительной пиротехнической смеси $\mathrm{NCP} / \mathrm{GnS}$ 97/3\% массой $~ 0,12$ г (мощность излучения 10 Вт, длительность импульса 1 - 2 мс). Как показали испытания, время срабатывания макета ЛКД составляло около 12 мкс (энергия инициирования $~ 120$ мкДж). В то же время макет ЛКД на основе заряда комплекса NCP без светочувствительной добавки удалось инициировать лучом лазерного диода только при длительности импульса более 2 мс (при энергии инициирования более 20 мДж).

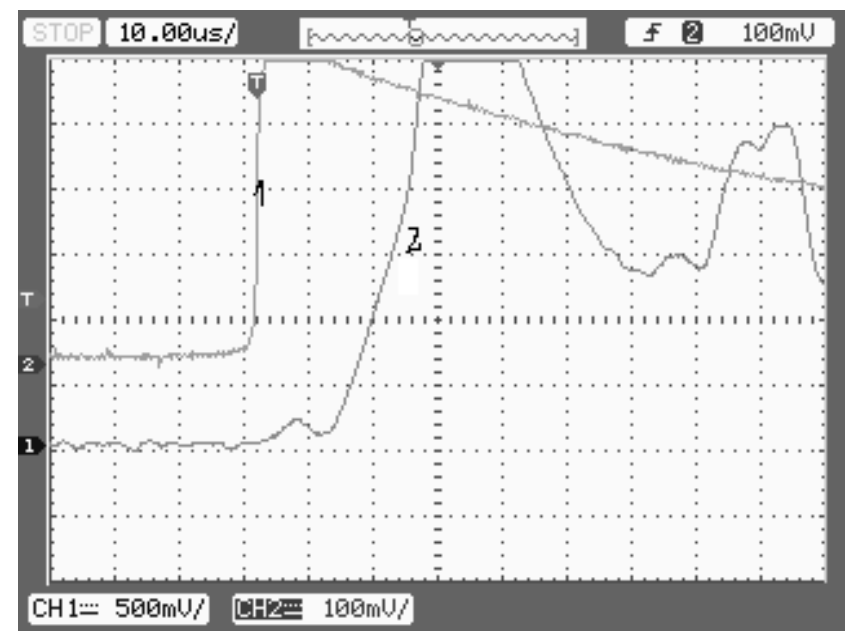

Рис. 2. Осциллограмма процесса лазерного инициирования заряда смеси NCP/GnS 97/3\%

1 - сигнал с измерительного световода;

2 - сигнал с фотодиода после разрушения оболочки (выход свечения)

Инициирующую способность макета ЛКД оценивали по пробитию свинцовой пластины. Суть метода заключалась в следующем: при дефлаграции отпечаток на свинцовой пластине отсутствует, а в случае перехода процесса горения в детонацию в пластине-свидетеле образовывалось отверстие. Дополнительно проводили испытания ЛКД на стальной плите, в которых оценивали эффективность изделий по глубине отпечатка $[15,16]$.

Как показали эксперименты, луч лазерного диода зажигал заряд светочувствительной пиротехнической смеси NCP/GnS 97/3\%. Смесь инициировала взрыв заряда комплекса NCP, который, в свою очередь, вызвал детонацию заряда вторичного ЭМ. Продукты детонации последнего пробили отверстие в свинцовой пластине.

Таким образом, добавка $3 \% \mathrm{GnS}$ к комплексу NCP дала возможность создать высокоэффективную пиротехническую смесь, которая требовала примерно на порядок меньше лазерной энергии для инициирования полноценной детонации макета ЛКД по сравнению с аналогичной моделью ЛКД, в которой использовали индивидуальный комплекс NCP, имевший равную с пиротехнической смесью дисперсность.

Наблюдаемый эффект повышения чувствительности комплекса NCP к лазерному инициированию является новым и механизм его возникновения требует последовательного анализа.

В работе [17] было изучено влияние добавок $\mathrm{GnS}$ на порог разложения комплекса NCP при воздействии фемтосекундным лазерным излучением с длиной волны 1554 нм (длительность импульса 100 фс $\left(1 \cdot 10^{-13} \mathrm{c}\right)$ и частотой импульсов 76 МГц). Длина волны излучения 1554 нм - это область селективного поглощения лазерной энергии комплексом NCP. Было показано, что зависимость носит экстремальный характер, причем минимальный порог инициирования вспышки на участке поверхности образца комплекса NCP зафиксирован при введении 3 масс. \% графена, величина наблюдаемого порога зажигания которого примерно на порядок ниже, чем у исходного комплекса NCP. Интенсивность лазерного излучения в экстремуме составляла $\sim 0,15 \mathrm{BT}^{*} \mathrm{мм}^{-2}$. Авторы работы предложили и подтвердили расчетами тепловой механизм воспламенения смесей $\mathrm{NCP} / \mathrm{GnS}$ [17]. В рамках предложенной модели роль добавки $\mathrm{GnS}$ сводилась к введению в систему абсолютно черного тела, обеспечивающего рост эффективного коэффициента оптического поглощения лазерного излучения и, тем самым, снижающего порог воспламенения светочувствительного ЭМ. Композиционный инициатор ( $\mathrm{GnS} /$ комплекс $\mathrm{NCP}$ ) рассмотрен авторами как простая механическая смесь двух порошков.

Однако нельзя исключить, что при смешении комплекса NCP и графена $\mathrm{GnS}$ происходит взаимодействие между компонентами смеси, инициируемое полем ультразвука. Сравнение ИК-спектров комплекса 1 и пиротехнической смеси $\mathrm{NCP} / \mathrm{GnS}$ подтвердило сделанное предположение. Так, в ИКспектрах смеси NCP/GnS в области «отпечатков пальцев» наблюдали значительные изменения по сравнению с ИК-спектром комплекса NCP: вместо полосы $1350 \mathrm{~cm}^{-1}$, отнесенной к деформационным колебаниям $\mathrm{NH}_{3}$ группы, появились две новые полосы $1398 \mathrm{~cm}^{-1}$ и $1384 \mathrm{~cm}^{-1}$, полоса поглощения $1320 \mathrm{~cm}^{-1}$, отнесенная к колебаниям нитрогруппы - $\mathrm{NO}_{2}$ и тетразольного кольца приобрела дублетный характер. Такие изменения в ИК-спектре смеси подтверждают взаимодействие поверхностей ком- 
плекса NCP и графена GnS. Причем во взаимодействии участвуют как координированные молекулы аммиака, так и нитротетразолатный лиганд. Обнаруженное взаимодействие поверхностей компонентов пиротехнической смеси, очевидно, стало одной из причин значительной депрессии (более чем на $\left.30^{\circ} \mathrm{C}\right)$ температуры начала разложения $\left(T_{\text {нр. }}\right)$ пиротехнических составов NCP/GnS по сравнению с индивидуальным комплексом NCP [18].

Следовательно, композиции $\mathrm{GnS}$ с комплексом NCP представляют собой не механические смеси компонентов, а ассоциаты, характеризующийся собственными термохимическими параметрами, что не учитывала тепловая модель зажигания смесей NCP/GnS, предложенная в работе [17].

Необходимо обратить внимание, что графен как малодефектный кристалл при комнатной температуре способен проводить электроны быстрее многих других веществ [19]. Кроме того, 2D листы GnS обладают свойством формировать поток электронов под воздействием потока фотонов $[20,21]$. Способность графена $\mathrm{GnS}$ генерировать потоки электронов имеет принципиальное значение для объяснения снижения порога лазерного инициирования для пиротехнической смеси $\mathrm{NCP} / \mathrm{GnS}$, поскольку $\mathrm{GnS}$ может выступать как источник электронов, приводящих к взрывчатому разложению ЭМ по механизму электрического пробоя.

Данная описательная модель дает возможность качественно объяснить результаты действия графена GnS на подрыв комплекса NCP как при действии сильноточного пучка электронов, так и при облучении когерентным излучением разной длительности и длины волны.

\section{ЛИТЕР АТ УРА}

1. Brink T. Green energetic materials. John Wiley \& Sons Ltd. 2014. P. 304.

2. Илюшин М.А., Котомин А.А., Душенок С.А. Энергонасыщенные металлокомплексы. Химическая физика. 2019. T. 38. № 2. C. 24-44.

3. Ahmad S.R., Cartwright M. Laser Ignition of Energetic Materials. Chichester: John Wiley \& Sons, 2015.

4. Handbook of Ecomaterials /Eds. Martínez L.M.T., Kharissova O.V., Kharisov B.I., Volum 5, Part XVII, Ecomaterials with Special Properties, 138 Eco-friendly Energetic Substances for Initiation Devices, Contributors: M.A Ilyushin, I.V. Shugalei, Springer Nature Switzerland AG, Cham. 2019. 3433. DOI: 10.1007/978-3-319-68255-6_46.

5. Sunahara G.I., Lotufo G., Kuperman R.G., Hawari J. Ecotoxicology of Explosives. London / New York: Taylor and Francis Group. 2009. P. 336.

6. Смирнов А.В., Федотов С.А., Агеев М.В., Ведерников Ю.Н., Илюшин М.A. Поиск перспективных взрывчатых веществ для средств инициирования в ряду тетразолатных амминатов кобальта (III). Боеприпасы и высокоэнергетические конденсированные системы: научно-технический журнал. 2018. № 1. С. 31-36.
Какой из предложенных механизмов инициирования смесей $\mathrm{NCP} / \mathrm{GnS}$ реализуется на практике в каждом конкретном случае: тепловой или электрического пробоя, предстоит выяснить в дальнейших исследованиях.

\section{ВЫВОДЫ}

1. Анализ литературы и всей совокупности полученных в ходе работы экспериментальных данных позволяет считать наиболее вероятным утверждение, что инициирование взрывчатого разложения разработанного пиротехнического состава NCP/GnS происходит вследствие возникновения фототока в 2D пластинах многослойного графена $\mathrm{GnS}$ под воздействием когерентного лазерного излучения с длиной волны 976 нм (явление фотовольтаики).

2. Проведены испытания макетов лазерного капсюля-детонатора (ЛКД) на базе пиротехнического состава $\mathrm{NCP} / \mathrm{GnS}$ при соотношении компонентов (97/3) масс. \%, который требует для инициирования макета ЛКД примерно в 200 раз меньше лазерной энергии, чем для инициирования макета ЛКД, содержавшего в качестве светочувствительного вещества заряд только комплекса NCP.

3. Результаты работы могут служить основой для разработки безопасных, надежных и экологичных ЛКД нового поколения для применения в горнодобывающей, нефтегазовой промышленности и других отраслях народного хозяйства.

Авторы заявляют об отсутствии конфликта интересов, требующего раскрытия в данной статье.

Исследование выполнено при финансовой поддержке РФФИ в рамках научных проектов 17-03-00566 и 18-29-24129мк.

\section{REFERENCES}

1. Brink T. Green energetic materials. John Wiley \& Sons Ltd. 2014. P. 304.

2. Ilyushin M.A., Kotomin A.A., Dushenok S.A. Energy-Saturated Metal Complexes. Russian Journal of Physical Chemistry B. 2019. V. 13. N 1. P. 119-138. DOI: 10.1134/S1990793119010238.

3. Ahmad S.R., Cartwright M. Laser Ignition of Energetic Materials. Chichester: John Wiley \& Sons, 2015.

4. Handbook of Ecomaterials /Eds. Martínez L.M.T., Kharissova O.V., Kharisov B.I., Volum 5, Part XVII, Ecomaterials with Special Properties, 138 Eco-friendly Energetic Substances for Initiation Devices, Contributors: M.A. Ilyushin, I.V. Shugalei, Springer Nature Switzerland AG, Cham. 2019. 3433. DOI: 10.1007/978-3-319-68255-6_46.

5. Sunahara G.I., Lotufo G., Kuperman R.G., Hawari J. Ecotoxicology of Explosives. London / New York: Taylor and Francis Group. 2009. P. 336.

6. Smirnov A.V., Fedotov S.A., Ageev M.V., Vedernikov Yu.N., Ilyushin M.A. Search of promising explosives for initiation agents in the series of tetrazolate amminates of cobalt (III). Boepripasy i vysokoenergeticheskie kondensirovannye sistemy: nauchno-tachnicheskii zhurnal. 2018. № 1. P. 31-36. (in Russian). 
7. Savenkov G.G., Ilyushin M.A. Initiation of explosive transitions in energy-saturated cobalt salt and nanosized carbonic additives compounds by means high-current electron beam. Proceedings of the 19-th Seminar "New trends in research of energetic materials" Pardubice. Czech Republic. April 20-22. 2016. Part 1I. P. 891-895.

8. Савенков Г.Г., Морозов В.А., Илюшин М.А., Оськин И.А., Брагин B.A., Козлов А.С. Влияние наноразмерных форм углерода на свойства и восприимчивость к импульсному пучку электронов энергонасыщенной соли кобальта. Журнал технической физики. 2017. Т. 87. Вып. 11. С. 1701-1706.

9. Савенков Г.Г., Морозов В.А., Илюшин М.А., Каи М.М. Графен как сенсибилизирующая добавка в энергонасыщенную соль кобальта для усиления воздействия сильноточного электронного пучка. Письма в журнал технической физики. 2018. Т. 44. № 12. С. 39-44.

10. Zhang, X., Hikal W., Zhang Y., Bhattcacharia S., Li L., Wang S.R., Weeks B.L. Direct laser initiation and improved thermal stability of nitrocellulose/graphene oxide nanocomposites. Applied Physics Letters. 2013. 102(14). P. 5428. DOI: 10.1063/1.4801846.

11. Liu C.J., Li X.D., Li R., Yang Q., Zhang H.P., Yang B., Yang G.C. Laser ignited combustion of graphene oxide/nitrocellulose membrane for solid propellant micro thruster and solar water distillation. Carbon. 2020. 166. P. 138-147. DOI: 10.1016/j.carbon.2020.05.014.

12. Li X.D., Huang B., Li R., Zhang H.P., Qin W.Z., Qiao Z.Q., Liu Y.S., Yang G.C. Laser-Ignited Relay-Domino-Like Reactions in Graphene Oxide/CL-20 Films for High-Temperature Pulse Preparation of Bi-Layered Photothermal Membranes. Small. 2019. P. 1900338. DOI: $10.1002 / \mathrm{smll} .201970107$.

13. Voznyakovskii A.P., Neverovskava A.Yu., Otvalko Ja.A., Gorelova E.V., Zabelina A.N. Facile synthesis of 2D carbon structures as a filler for polymer composites. Nanosystems: Physics, Chemistry, Mathematics. 2018. V. 9. N 1. P. 125-128. DOI 10.17586/2220-8054-2018-9-1-125-128.

14. Matyáš R, Pachman J. Primary explosives. Heidelberg: Springer. 2013. DOI: 10.1007/978-8-642-28436-6.

15. Zeman S., Jungova M. Technology of Explosives. Pardubice: University of Pardubice. 2019.

16. Klapötke T.M. Chemistry of High-Energy Materials. $4^{\text {th }}$ ed. Berlin/Boston: Walter de Gruyter GmbH. 2017. DOI: 9783110536515-202.

17. Kuzmenko A.V., Tverjanovich A.S., Ilyushin M.A., Tveryanovich $Y u . S$. The effect of the concentration of high-absorbing inclusions on the laser initiation threshold of energetic materials: model and experiment. Journal of Energetic Materials. 2019. V. 37. N 4. P. 420-432. DOI: 10.1080/07370652.2019.1630028.

18. Ilyushin M.A., Vozniakovskii A.P., Shugalei I.V., Shustrova O.P., Kozlov A.S., Savenkov G.G., Vozniakovskii A.A., Tverianovich A.S. Mixtures of pentaammine(5-nitrotetrazolato - N2)cobalt (III) perchlorate and nanocarbon. Proceedings of the 22-th Seminar "New trends in research of energetic materials" Pardubice. Czech Republic. April 10-12. 2019. Part 1I. P. 431-434.

19. Wilson M. Electrons in Atomically Thin Carbon Sheets Behave Like Massless Particles. Physics Today. 2006. V. 59. No.1. P. 21-23. DOI: 10.1063/1.2180163.

20. Katsnelson M.I., Novoselov K.S. Graphene: New bridge between condensed matter physics and quantum electrodynamics. Solid State Communications. 2007. V. 143. P. 3-13. DOI: $10.1016 /$ j.ssc.2007.02.043.

21. Елеикий А.В., Зичерман В.Ю., Кобзев Г.А. Графен в солнечной энергетике. Российские нанотехнологии. 2015. Т. 10. № 3-4. C. 16-25.
7. Savenkov G.G., Ilyushin M.A. Initiation of explosive transitions in energy-saturated cobalt salt and nanosized carbonic additives compounds by means high-current electron beam. Proceedings of the 19-th Seminar "New trends in research of energetic materials" Pardubice. Czech Republic. April 20-22. 2016. Part 1I. P. 891-895.

8. Savenkov G.G., Morozov V.A., Ilyushin M.A., Oskin I.A., Bragin V.A., Kozlov A.S. Influence of Nanosized Carbon Forms on the Properties and Susceptibility of Energy-Saturated Cobalt Salt to a Pulsed Electron Beam. Technical Physics. 2017. V. 62. N 11. P. 1703-1708. DOI: 10.1134/S1063784217110263.

9. Savenkov G.G., Morozov V.A., Ilyushin M.A., Kats V.M. Graphene As a Sensitizing Additive to High-Energy Cobalt Salt for Enhanced Initiation by a High-Current Electron Beam. Technical Physics Letters. 2018. V. 44. N 6. P. 522-524. DOI: 10.1134/S1063785018060275.

10. Zhang, X., Hikal W., Zhang Y., Bhattcacharia S., Li L., Wang S.R., Weeks B.L. Direct laser initiation and improved thermal stability of nitrocellulose/graphene oxide nanocomposites. Applied Physics Letters. 2013. 102(14). P. 5428. DOI: $10.1063 / 1.4801846$.

11. Liu C.J., Li X.D., Li R., Yang Q., Zhang H.P., Yang B., Yang G.C. Laser ignited combustion of graphene oxide/nitrocellulose membrane for solid propellant micro thruster and solar water distillation. Carbon. 2020. 166. P. 138-147. DOI: 10.1016/j.carbon.2020.05.014.

12. Li X.D., Huang B., Li R., Zhang H.P., Qin W.Z., Qiao Z.Q., Liu Y.S., Yang G.C. Laser-Ignited Relay-Domino-Like Reactions in Graphene Oxide/CL-20 Films for High-Temperature Pulse Preparation of Bi-Layered Photothermal Membranes. Small. 2019. P. 1900338. DOI: 10.1002/smll.201970107.

13. Voznyakovskii A.P., Neverovskaya A.Yu., Otvalko Ja.A., Gorelova E.V., Zabelina A.N. Facile synthesis of 2D carbon structures as a filler for polymer composites. Nanosystems: Physics, Chemistry, Mathematics. 2018. V. 9. N 1. P. 125-128. DOI 10.17586/2220-8054-2018-9-1-125-128.

14. Matyáś $R$, Pachman J. Primary explosives. Heidelberg: Springer. 2013. DOI: 10.1007/978-8-642-28436-6.

15. Zeman S., Jungova M. Technology of Explosives. Pardubice: University of Pardubice. 2019.

16. Klapötke T.M. Chemistry of High-Energy Materials. $4^{\text {th }}$ ed. Berlin/Boston: Walter de Gruyter GmbH. 2017. DOI: 9783110536515-202.

17. Kuzmenko A.V., Tverjanovich A.S., Ilyushin M.A., Tveryanovich $Y u$.S. The effect of the concentration of high-absorbing inclusions on the laser initiation threshold of energetic materials: model and experiment. Journal of Energetic Materials. 2019. V. 37. N 4. P. 420-432. DOI: 10.1080/07370652.2019.1630028.

18. Ilyushin M.A., Vozniakovskii A.P., Shugalei I.V., Shustrova O.P., Kozlov A.S., Savenkov G.G., Vozniakovskii A.A., Tverianovich A.S. Mixtures of pentaammine(5-nitrotetrazolato - N2)cobalt (III) perchlorate and nanocarbon. Proceedings of the 22-th Seminar "New trends in research of energetic materials" Pardubice. Czech Republic. April 10-12. 2019. Part 1I. P. 431-434.

19. Wilson M. Electrons in Atomically Thin Carbon Sheets Behave Like Massless Particles. Physics Today. 2006. V. 59. No.1. P. 21-23. DOI: 10.1063/1.2180163.

20. Katsnelson M.I., Novoselov K.S. Graphene: New bridge between condensed matter physics and quantum electrodynamics. Solid State Communications. 2007. V. 143. P. 3-13. DOI: $10.1016 /$ j.ssc.2007.02.043.

21. Eletskiy A.V., Zitserman V.Yu., Kobzev G.A. Graphene in solar energy. Rossiiskie nanotechnologii. 2015. V.10. № 3-4. P. 16-25 (in Russian).

Поступила в редакиию (Received) 01.08.2021

Принята к опубликованию (Accepted) 03.09.2021 